\title{
Real-time Audio \& Video Transmission System Based on Visible Light Communication
}

\author{
Yingjie He, Liwei Ding, Yuxian Gong, Yongjin Wang* \\ Institute of Communication Technology, Nanjing University of Posts and Telecommunications, Nanjing, China \\ Email: *wangyj@njupt.edu.com
}

Received 2013

\begin{abstract}
With the increasing popularity of solid sate lighting devices, Visible Light Communication (VLC) is globally recognized as an advanced and promising technology to realize short-range, high speed as well as large capacity wireless data transmission. In this paper, we propose a prototype of real-time audio and video broadcast system using inexpensive commercially available light emitting diode (LED) lamps. Experimental results show that real-time high quality audio and video with the maximum distance of $3 \mathrm{~m}$ can be achieved through proper layout of LED sources and improvement of concentration effects. Lighting model within room environment is designed and simulated which indicates close relationship between layout of light sources and distribution of illuminance.
\end{abstract}

Keywords: Visible Light Communications; LED; Real-Time Video and Audio Broadcast System; Light Source Arrangement; Illuminance Distribution

\section{Introduction}

Visible Light Communication (VLC) was first proposed in 2004 by Toshihiki Komine and has progressed rapidly ever since with the development of solid state light sources, especially light emitting diodes (LEDs) [1]. The great popularity of VLC owes largely to the advantages of LED such as high brightness, low cost, small size, low power consumption, long lifetime and low heat radiation. VLC explores the unregulated visible light portion of the electromagnetic spectrum and acts as a supplement rather than substitute of established RF systems. With complementary strengths of both technologies, excess capacity demands of RF channels can be off-loaded to VLC networks, which enable users to seamlessly access to the Internet while keeping high Qos levels and avoiding network congestions and delays. The concept of a fullduplex multi-access system for LED-based wireless communications is proposed by Nakagawa laboratories [1]. Simplex and duplex transceiver prototypes have been demonstrated in [2] which also prove the effectiveness of replacing existing illumination systems with LED lighting devices. With $300 \mathrm{THz}$ of bandwidth available for VLC, multi- gigabit-per-second data rates could be provided over short distances, for example, using array of LEDs in a multiple-input multiple-output (MIMO) system $[3,4]$. Besides wireless connectivity in home environment, combining the function of illumination and

${ }^{*}$ Corresponding author. transmission is also attractive in specific scenarios where healthy, secured and non-interfered communication is necessary, such as hospital, underground mine, underwater, airplane, etc.

In this paper, we develop a VLC prototype with large increase in transmission distance and improvement in channel capacity. The optical link which performs as the substitute of connector wire can be widely applied in video conference, real-time video frequency monitoring, smart traffic system and various practical scenarios where illumination and data transmission is in joint demand to serve modern daily life. MATLAB program is used to simulate the illuminance distribution for two practical light source deployments.

The rest of the paper is organized as follows: In section 2, a prototype of the VLC real-time video/audio transmission system is presented. The experimental result is shown and analyzed in section 3. Section 4 illustrates the correlation between light source layout and illuminance distribution based on MATLAB simulation within a model room. Finally, Section 5 concludes the article.

\section{VLC System Description}

Figure 1 shows the block diagram of the real-time video and audio broadcast system, which consists of a VLC wireless LOS link with two optical channels.

VLC is free space optical communication, and line of sight (LOS) is the common link between two points in optical wireless communication system, where the trans- 
mitter directs the visible light beam in a straight and unobstructed path to the receiver [5]. Intensity modulation and direct detection are adopted by the prototype for data transmission. The system is constructed as Figure 2. The light emitter is a simple, off-the shelf LED obtained from Cree (XLamp MX-6) which provides a luminous flux of $107 \mathrm{~lm}$ at the current of $350 \mathrm{~mA}$. A Reflection cup is mounted on each LED for the purpose of gaining light that is more parallel and concentrated. Video and audio signal captured by video camera are amplified by a self-designed amplifier and then superimposed onto two LED lamps respectively by the aid of a bias-T circuit. Thus, the output light rays changes in intensity corresponding to the variation in signal, which however is insensitive to human eyes due to the rapid frequency response of LED devices. The distance between two LEDs was about $10 \mathrm{~cm}$ in order to avoid mutual interference caused by light sources. The transmitter circuit is given in Figure 3. At the receiver, two highly sensitive Si PIN photodiodes (Hamamtsu S6968) are used to detect light transmitted over two separate optical channels. And the directionality of the PDs is required to be aligned with the most intense portion of the emitted light beams. After detection, optical signals are converted into photo electric current proportional to the variation of incident light which then is amplified and filtered by a low pass filter (LPF). The receiver circuit is shown in Figure 4. An audio and video capture module with USB interface is designed to convert the received analog signal into digital signal [6]. Finally, the video and audio are played real time on the screen of a video editor for further processing such as simple cutting, filtering or storing. In the prototype, transmission distance of nearly $2 \mathrm{~m}$ can be achieved without any light focusing measures and can be increased to $3 \mathrm{~m}$ with a focusing lens inserted between the transmitter and receiver.

\section{Results and Analysis}

To test the system performance, we have transmitted a video signal captured by a video camera. Video and audio signals were extracted from the AV output port of the camera. Through optical transmission, high quality video and audio were recovered and played on the laptop screen by the aid of self-designed ADC module with USB 2.0 interface. High-speed rendering, wireless transmission and real time performance mean less time waiting and more time creating. Figure 5 shows the waveforms of video signal before and after transmission over the optical channel. And the picture shown in Figure 6

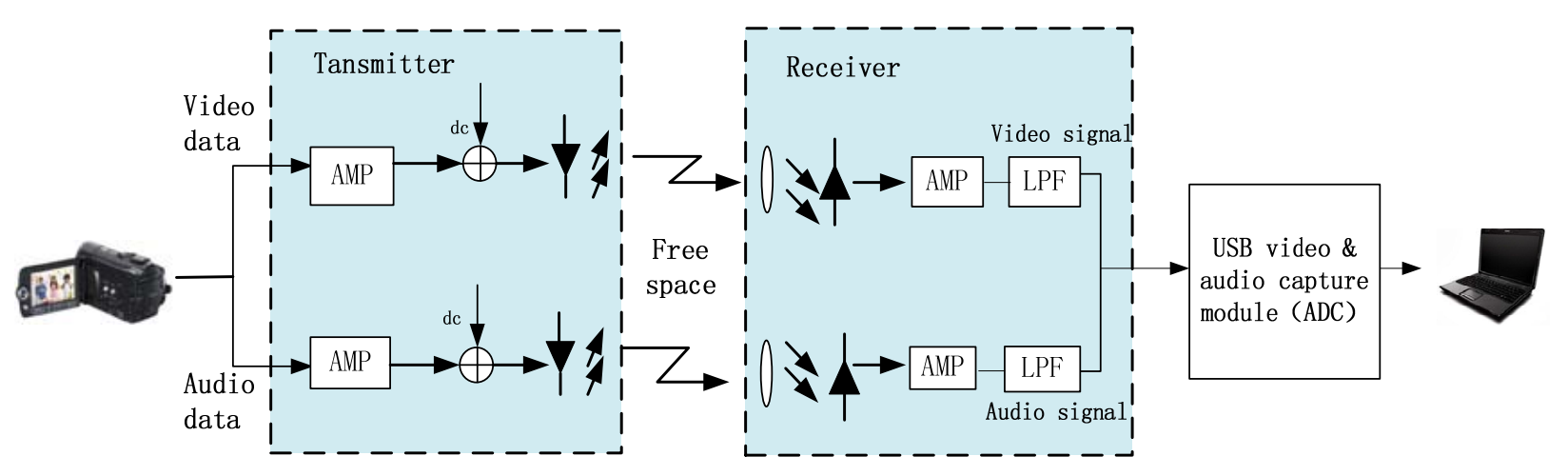

Figure 1. Block diagram of real-time video/audio VLC transmission system.

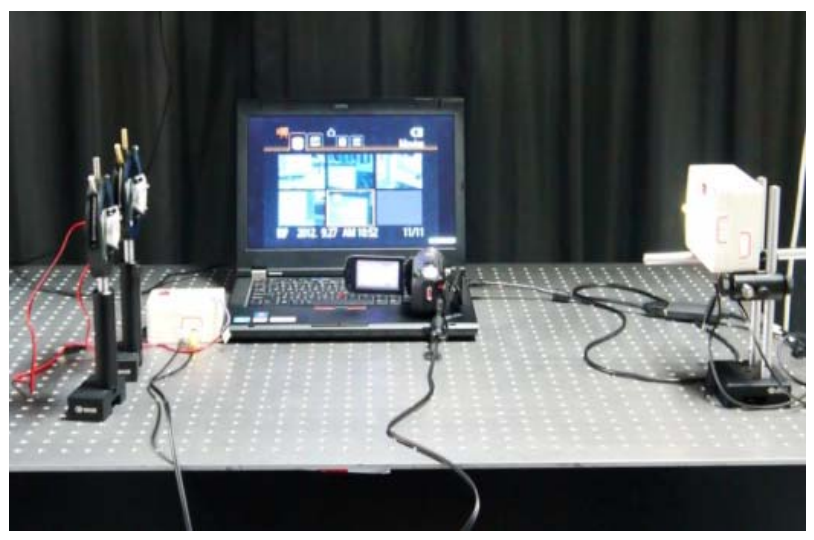

Figure 2. Picture of the real-time video/audio transmission prototype using LEDs.

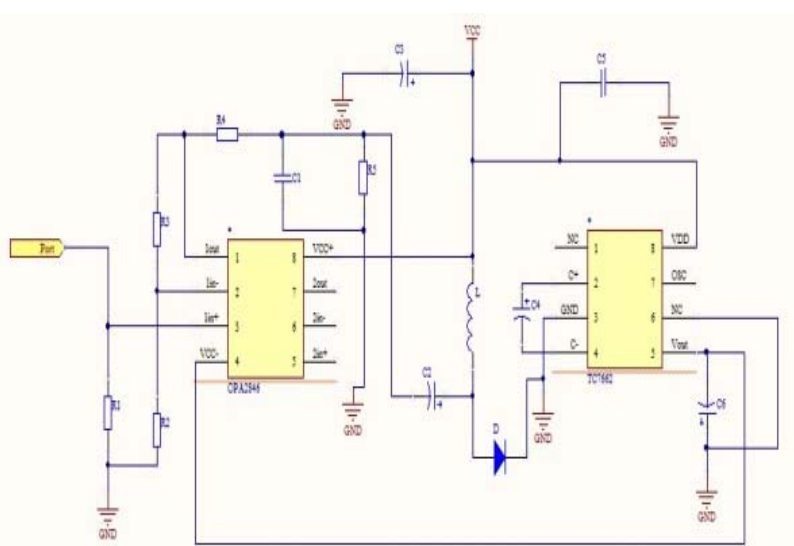

Figure 3. Transmitter circuit. 


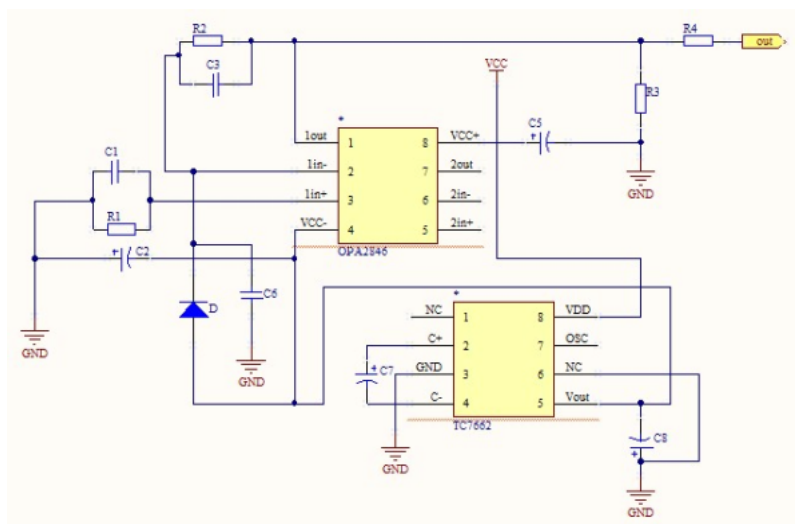

Figure 4. Receiver circuit.

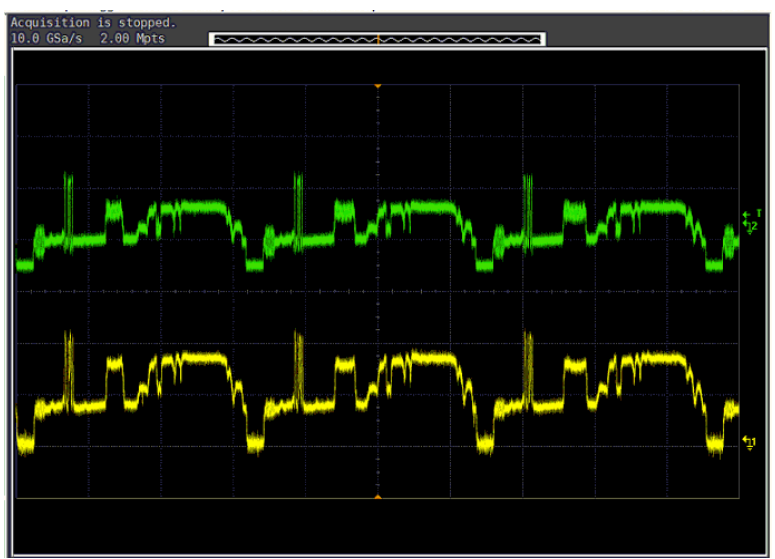

Figure 5. Experimental test of VLC prototype, received signal at the distance of $2 \mathrm{~m}$, emitted (up) and received signal (bottom).

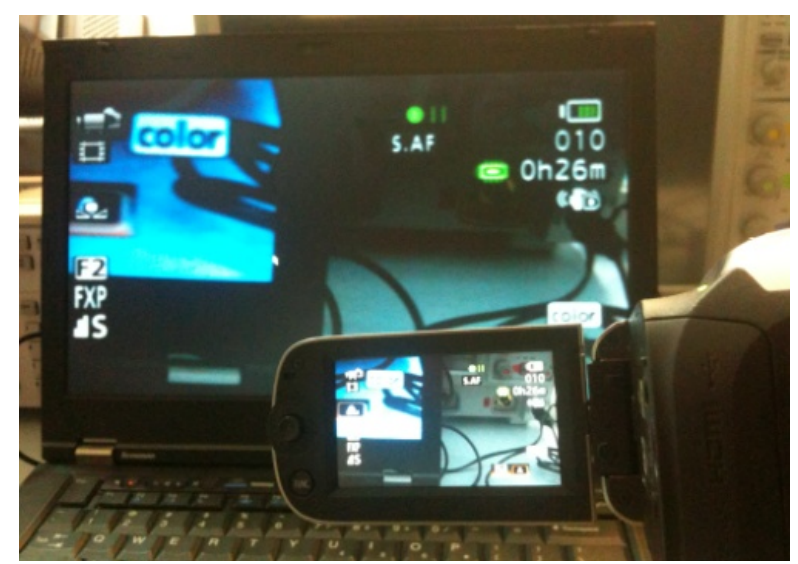

Figure 6. Comparison between original image and received image.

clearly indicates that signal transmitted by optical channel can be recovered real time at $2 \mathrm{~m}$ without lens through the air in the laboratory. Some distortions still exist comparing the original to the received image which maybe results from the process of photoelectric conversion, signal amplification or the introduction of background noise.
To improve the system performance, analog signal from web camera should be converted into digital signal before optical wireless transmission.

\section{Modeling and Designing of an Indoor VLC System}

The final objective of VLC development is the application of off-the-shelf LEDs in home environment wireless network to satisfy the needs of both illumination and data transmission. The prototype presented above with single LED replaced by LED arrays, is capable of supporting lighting, data broadcast as well as monitoring. In the paper, we modeled a room with the size of $5 * 5 * 3(\mathrm{~m})$. To study the illuminance distribution of LED system in practical scenarios, two types of light source arrangements located on the ceiling were simulated and discussed as shown in Figure 7. The coordinate positions of LED arrays for square and round deployments are (1.05:0.1:1.55, 1.05:0.1:1.55, 3) and $\left(2.5+r^{*} \cos ((0: 1: 17+\right.$ $\left.0.5)^{*} \mathrm{pi} / 36\right), \quad 2.5-\mathrm{r}^{*} \sin \left((\mathrm{i}+0.5)^{*} \mathrm{pi} / 36\right), 3$ ) respectively. Here, 1.05:0.1:1.55 indicates that the value on coordinates increases with the step size of $0.1 \mathrm{~m}$, and $0: 1: 17$ means that the step size is assumed to be $1 \mathrm{~m}$. The value of $i$ is $1: 1: 18$ and the radius are set to be $R 1=2.15 \mathrm{~m}$ and $\mathrm{R} 2=2.35 \mathrm{~m}$. Assuming that radiation of LEDs conforms to the Lambertian pattern and light reflections on the wall are not considered. The horizontal light illuminance at the point $(\mathrm{x}, \mathrm{y}, \mathrm{z})$ is given by

$$
E(x, y, z)=\frac{I(0) \cos ^{m}(\phi)}{D^{2} \cdot \cos (\psi)}
$$

where, $I(0)$ is the center luminous intensity of LEDs, $\phi$ is the angle of irradiance, $\psi$ is angle of incidence, $D$ is the distance between the LED transmitter and the receiver, and $\mathrm{m}$ is the order of Lambertian emission given by

$$
m=-\ln 2 / \ln \left(\cos \varphi_{1 / 2}\right)
$$

where, $\varphi_{1 / 2}$ is the semi-angle at half power $[7,8]$. MATLAB simulation specifications are depicted in Table $\mathbf{1 .}$

The simulation of illuminance distribution for two different light source arrangements is shown in Figure 8. The minimum and maximum values for the square layout are $310.59 \mathrm{~lx}$ and $823.42 \mathrm{~lx}$ respectively. Figure 8(b)
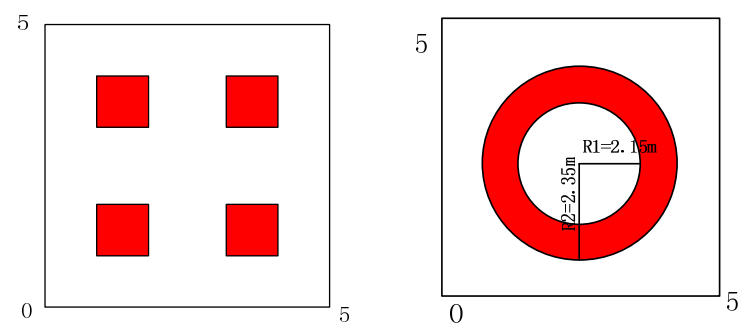

Figure 7. Square array (Left) \& Round array (right). 
Table 1. Parameters for MATLAB simulation.

\begin{tabular}{lcc}
\hline \multicolumn{1}{c}{ Parameters } & Values(square) & Values(round) \\
\hline Room size & $5 * 5 * 3(\mathrm{~m})$ & $5 * 5 * 3(\mathrm{~m})$ \\
Number of LED arrays & $4 * 36$ & $4 * 36$ \\
Semi-angle at half power & $45^{\circ}$ & $45^{\circ}$ \\
Luminous intensity & $10(\mathrm{~cd})$ & $10(\mathrm{~cd})$ \\
Distance between LEDs & $10(\mathrm{~cm})$ & $20(\mathrm{~cm})$ \\
\hline
\end{tabular}

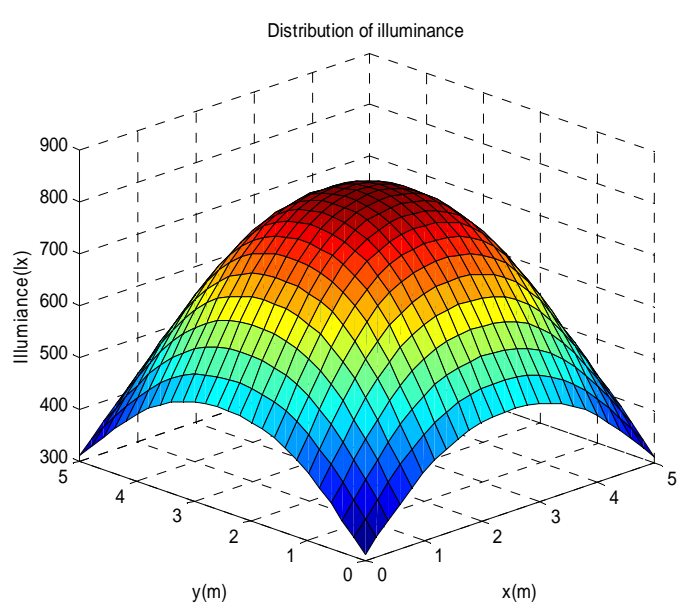

(a)

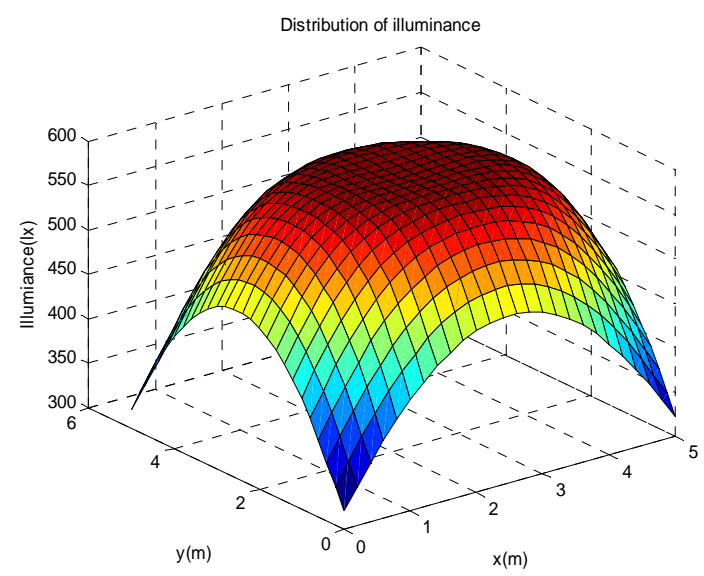

(b)

Figure 8. Illuminance distribution with the LED semi-angle of 45 degree (a) Illuminance distribution for $4 \times 4$ square deployment (b) Illuminance distribution for round deployment.

gives the simulation results for round deployment with the maximum value of $321.79 \mathrm{~lx}$ and minimum value of 592.92 lx. Considering that the lighting illumination requirements of the International Organization for Standardization is from 300lx to $1500 \mathrm{~lx}$ [7], the two arrangements of light sources proposed here can meet the joint demands of illumination and communication based on the simulation results. Furthermore, illuminance for round LED arrangement is more evenly distributed than that of the square arrangement judging from the simulation diagram, so that lighting effects for round deployment should be better in this case.

\section{Conclusions}

In this paper, we proposed a real-time video and audio broadcast prototype using commercial LED lamps. System design is illustrated in detail and experimental results are presented. It is shown that transmission of high quality video/audio images with the distance of $3 \mathrm{~m}$ can be achieved and improvements can be made by adding focusing lens between the transmitter and the receiver. Although distortions still exist comparing images before and after transmission, it demonstrates that high quality wireless optical transmission using LED is successful. Illuminance distribution is simulated and compared for two light sources arrangements in a model room. Future work will concentrate on real-time digital video/audio transmission and communication link that can be connected to a TCP/IP network [9].

\section{Acknowledgements}

This work is jointly supported by NSFC (11104147), Jiangsu 973 project (Bk2011027), and research project (NY211001, BJ211026).

\section{REFERENCES}

[1] Xin LIN, Kenichi IKAWA, Kazutoshi, HIROHASHI, Nakagawa Laboratories, Inc. "High-speed Full-Duplex Multiaccess System for LED-Based Wireless Communications Using Visible Light”, the 2nd International MultiConference on Engineering and Technological Innovation, 2009.

[2] T. D. C. Little, P. Dib, K. Shah, N. Barraford and B. Gallagher, "Using LED Lighting for Ubiquitous Indoor Wireless Networking," IEEE International Conference on Wireless and Mobile Computing, pp. 373-378, 2008. doi:10.1109/WiMob.2008.57

[3] Y. A. Alqudah and M. Kavehrad, "MIMO Characterization of Indoor Wireless Optical Link Using a Diffuse-Transmission Configuration," IEEE Transactions on Communications, Vol. 51, No. 9, pp. 1554-1560, 2003. doi:10.1109/TCOMM.2003.816945

[4] Z. Lubin, D. O’Brien, M. H. Le, G. E. Faulkner, L. Kyungwoo, J. Daekwang, O. YunJe and W. E. Tae, "High Data Rate Multiple Input Multiple Output (MIMO) Optical Wireless Communications Using White LED Lighting," IEEE Journal On Selected Areas In Communications, Vol. 27, No. 9, pp. 1654-1662, 2009. doi:10.1109/JSAC.2009.091215

[5] D. K. Son, E. B. Cho and C. G. Lee, "Demonstration of Visible Light Communication Link for Audio and Video Transmission,” Photonics Global Conference (PGC), pp. 
1-4, 2010. doi:10.1109/PGC.2010.5706094

[6] J. Rufo, J. Rabadan, F. Delgado, C. Quintana and R. Perez-Jimenez, "Experimental Evaluation of Video Transmission through LED Illumination Devices," Consumer Electronics, IEEE Transactions, Vol. 56, No. 3, pp. 1411-1416. doi:10.1109/TCE.2010.5606277

[7] H. Q. Nguyen, J. H. Choi, M. Kang, Z. Ghassemlooy, D. H. Kim, S. K. Lim, T. G. Kang and C. G. Lee, “A MATLAB-based Simulation Program for Indoor Visible Light Communication System,” Communication Systems Net- works and Digital Signal Processing, pp. 537-541, 2010.

[8] J. M. Kahn and J. R. Barry, "Wireless Infrared Communications,” Proceedings of the IEEE, pp. 265-298, 1997. doi:10.1109/5.554222

[9] J. Rufo, F. Delgado, C. Quintana, A. Perera, J. Rabadan and R. Perez-Jimmenez, "Visible Light Communication Systems for Optical Video Transmission," Microwave and Optical Technology Letters, Vol. 52, No. 7, pp. 1572-1576, 2010. doi:10.1002/mop.25236 